\title{
Creating Exact Bezier Representations of CST Shapes
}

\author{
David D. Marshall * \\ California Polytechnic State University, San Luis Obispo, CA 93407-0352, USA
}

\begin{abstract}
The paper presents a method of expressing CST shapes pioneered by Kulfan into standard Bezier curves and surfaces. Out of the seven standard shape classes identified by Kulfan as representable using CST curves, four of them, including airfoils, can be represented as Bezier curves exactly. For the other three, a convergent series expansion proves insufficient to achieve any practical accuracy, however these three classes are not the most commonly used of the CST classes. With the ability to express most common CST curves and surfaces as generic Bezier curves and surfaces, CST shapes can be used in CAD, solid modeling, meshing or any other geometry tool that does not explicitly support CST.
\end{abstract}

\section{Nomenclature}

$\begin{array}{ll}B_{i, n} & \mathrm{i}^{\text {th }} \text { Bernstein polynomial function of degree } n \\ C_{N_{2}}^{N_{1}} & \text { Class function function } \\ N_{1} & \text { First parameter in class function } \\ N_{2} & \text { Second parameter in class function } \\ p_{i} & \mathrm{i}^{\text {th }} \text { Control point of CST shape function } \\ \vec{p}_{i, j} & \text { Bezier surface control point } \\ S & \text { Shape function }\end{array}$

Conventions

CAGD Computer Aided Graphics Design

CST Class Shape Transformation

\section{Introduction}

$\mathrm{T}$

THERE has been an increased interest in using the class-shape transformation method (CST) ${ }^{1}$ to represent shapes in an aerodynamics context, especially airfoils. A lot of this attention is due to its use of relatively few number of parameters needed to express the shape. Equation (1) shows the general form of the CST method.

$$
\zeta(\xi)=C_{N_{2}}^{N_{1}} S(\xi)+\xi \Delta \zeta_{\text {t.e. }}
$$

where $N_{1}$ and $N_{2}$ are class parameters that determine the type of geometry to be represented, $S(\xi)$ is the shape function and $\Delta \zeta_{\text {t.e. }}$ is the trailing edge displacement. The $N_{1}$ and $N_{2}$ terms in (1) are collectively known as the class term and are sometimes represented as

$$
C_{N_{2}}^{N_{1}}(\xi)=\xi^{N_{1}}(1-\xi)^{N_{2}}
$$

Table 1 shows combinations for several common shapes and the corresponding class parameters. The shape function, $S(\xi)$, is typically shown as either a standard polynomial

$$
S(\xi)=\sum_{i=0}^{n} a_{i} \xi_{i}
$$

*Associate Professor, Aerospace Engineering Department, Senior Member AIAA. 
or as a Bezier curve

$$
S(\xi)=\sum_{i=0}^{n} B_{i, n}(\xi) p_{i}
$$

where $B_{i, n}(\xi)$ are the Bernstein polynomials and $p_{i}$ are the control points which represent the convex polygon containing the Bezier curve. Standard texts on computer aided graphics design (CAGD), such the one by Farin ${ }^{2}$ or Piegl and Tiller, ${ }^{3}$ will provide a number of useful properties of Bezier curves and Bernstein polynomials.

Table 1. Values of the class function terms for common geometries from Kulfan. ${ }^{1}$

\begin{tabular}{crcc} 
Index & Geometry Class & $N_{1}$ & $N_{2}$ \\
\hline 1 & Airfoil & $1 / 2$ & 1 \\
2 & Round Leading \& & $1 / 2$ & $1 / 2$ \\
& Trailing Edges & & \\
3 & Biconvex airfoil or & 1 & 1 \\
4 & Sharp Edges & & \\
5 & Low Drag Projectile & $3 / 4$ & $1 / 4$ \\
6 & Cone or Wedge & 1 & $\approx 0$ \\
7 & Rectangular Shape & $\approx 0$ & $\approx 0$
\end{tabular}

\section{Exact Transformation of CST Airfoils to Bezier Curves}

This section presents the mathematics behind the transformation of a CST airfoil, index 1 in table 1 , into Bezier curves with the use of approximations. While the standard CST representation is in an explicit form, it is $\zeta=f(\xi)$, it is necessary to convert it to a parameterized form. The standard CST representation, with $N_{1}$ and $N_{2}$ values substituted, is then represented as a pair of parametric equations for the $\xi$ - and $\zeta$-coordinates as

$$
\begin{aligned}
& \xi=t \\
& \zeta=\sqrt{t}(1-t) \sum_{i=0}^{n} B_{i, n}(t) p_{i}+t \Delta \zeta_{\text {t.e. }}
\end{aligned}
$$

To start, the shape function, which is expressed as an order $n$ Bezier curve, can be converted to a standard polynomial, i.e. with monomial basis functions. The following relations can be found in any thorough reference on Bezier curves, such as Piegl and Tiller. ${ }^{3}$

$$
\begin{aligned}
S(t) & =\sum_{i=0}^{n} B_{i, n}(t) p_{i} \\
& =\sum_{i=0}^{n} a_{i} t^{i} \\
\text { where } \quad a_{j} & =\sum_{i=0}^{j}\left(\begin{array}{l}
n \\
j
\end{array}\right)\left(\begin{array}{l}
j \\
i
\end{array}\right)(-1)^{j-i} p_{i}
\end{aligned}
$$

Now the $\zeta$-coordinate equation is

$$
\zeta=\sqrt{t}(1-t) \sum_{i=0}^{n} a_{i} t^{i}+t \Delta \zeta_{\mathrm{t} . \mathrm{e}}
$$


Next, the $N_{2}$ term of the class function can be combined with the shape function to obtain a polynomial of order $n+1$

$$
\zeta=\sqrt{t}\left[a_{0}+\sum_{i=1}^{n}\left(a_{i}-a_{i-1}\right) t^{i}-a_{n} t^{n+1}\right]+t \Delta \zeta_{\text {t.e. }}
$$

What is left is a polynomial in $t$ multiplied by a non-polynomial term, $\sqrt{t}$. If equation (9) can be expressed as a polynomial, then it can be converted to a Bezier curve using the inverse of equation (9). Introducing the parameter substitution of $t \equiv s^{2}$ does convert the $\xi$ - and $\zeta$-coordinate expressions to polynomials

$$
\begin{aligned}
& \xi=s^{2} \\
& \zeta=s\left[a_{0}+\sum_{i=1}^{n}\left(a_{i}-a_{i-1}\right) s^{2 i}-a_{n} s^{2(n+1)}\right]+s^{2} \Delta \zeta_{\text {t.e. }}
\end{aligned}
$$

Multiplying through by $s$ and simplifying yields

$$
\begin{aligned}
\xi & =s^{2} \\
\zeta & =a_{0} s+s^{2} \Delta \zeta_{\text {t.e. }}+\sum_{i=1}^{n}\left(a_{i}-a_{i-1}\right) s^{2 i+1}-a_{n} s^{2 n+3} \\
& =\sum_{i=0}^{2 n+3} b_{i} s^{i} \\
\text { where } \quad b_{i} & = \begin{cases}\Delta \zeta_{\text {t.e. }} & \text { if } i=2 \\
0 & \text { if } i \text { even and } i \neq 2 \\
a_{0} & \text { if } i=1 \\
-a_{n} & \text { if } i=2 n+3 \\
a_{i}-a_{i-1} & \text { otherwise }\end{cases}
\end{aligned}
$$

The final step in the process is converting the polynomials back to a Bezier curve using the following relation that converts a degree $m$ monomial basis polynomial with coefficients $d_{j}$ into a Bezier representation with control points $q_{i}$

$$
q_{i}=\frac{1}{\left(\begin{array}{c}
m \\
i
\end{array}\right)} \sum_{j=0}^{i} \begin{gathered}
m-j \\
i-j
\end{gathered} d_{j}
$$

In using equation (12), both the $\xi$ - and $\zeta$-coordinate expressions must be transformed. Thus instead of the one-dimensional control points that the CST formulation uses, the transformed bezier curve representation has two-dimensional control points and can be expressed as (with $m=2 n+3$ )

$$
\begin{aligned}
\vec{\xi} & =\left[\begin{array}{l}
\xi \\
\zeta
\end{array}\right]=\sum_{i=0}^{m} \vec{d}_{i} t^{i}=\sum_{i=0}^{m} B_{i, m}(t) \vec{q}_{i} \\
\text { where } \quad \vec{d}_{i} & =\left[\begin{array}{l}
c_{i} \\
b_{i}
\end{array}\right] \quad \text { and } \quad c_{i}= \begin{cases}1 & \text { if } i=2 \\
0 & \text { otherwise }\end{cases}
\end{aligned}
$$

Equation (13) represents an exact, standard Bezier curve representation of a CST airfoil. This curve can then be used in CAD, solid modeling, meshing or any other geometry tool that does not explicitly support CST airfoils.

Figure 1 shows a sample calculation of the corresponding Bezier curve for an $8^{\text {th }}$ order CST representation of an upper surface of an airfoil using the above relations.

\section{Application to Other CST Shapes}

While most of the uses of CST in the literature are for airfoils, the other classes in CST are also of interest. For the typical geometry classes from table 1, each one can either be represented exactly as a Bezier curve or approximated using a convergence series expansion for the class function. 


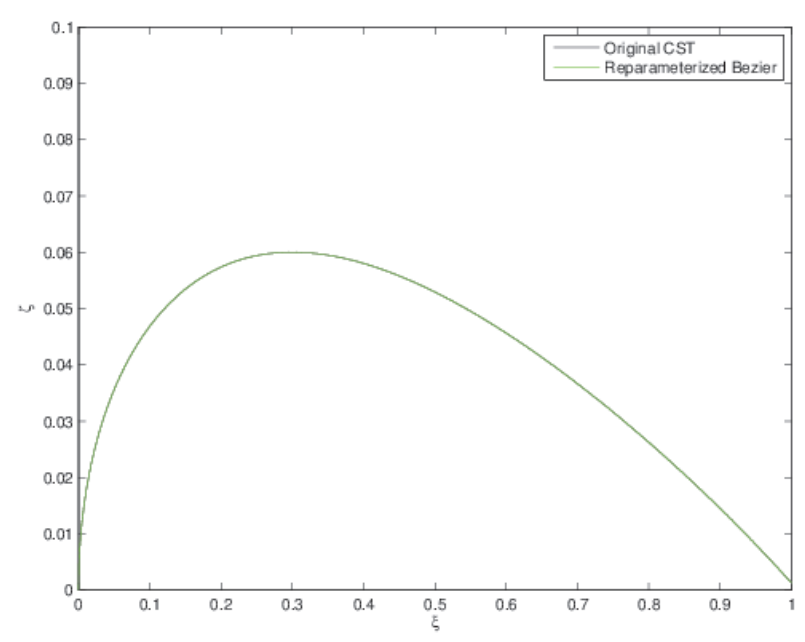

(a) Curves

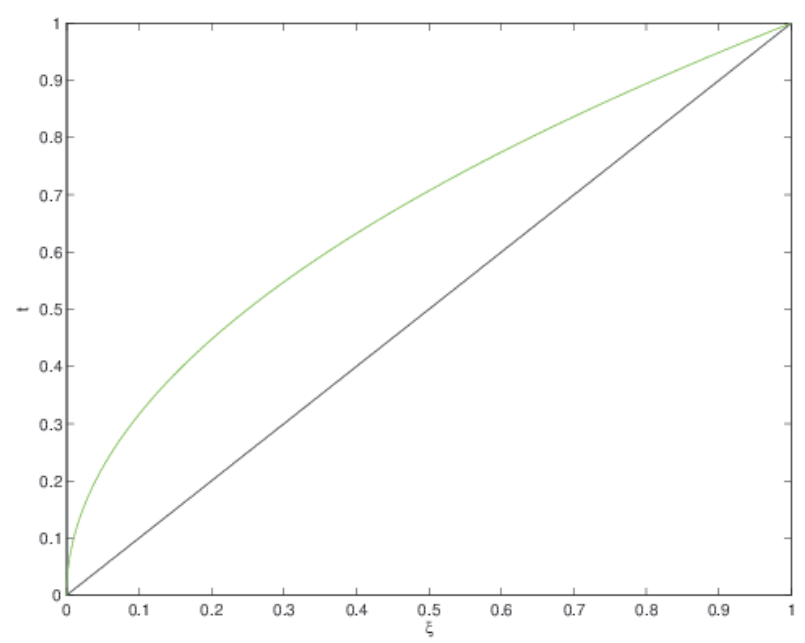

(c) $\xi$-coordinates

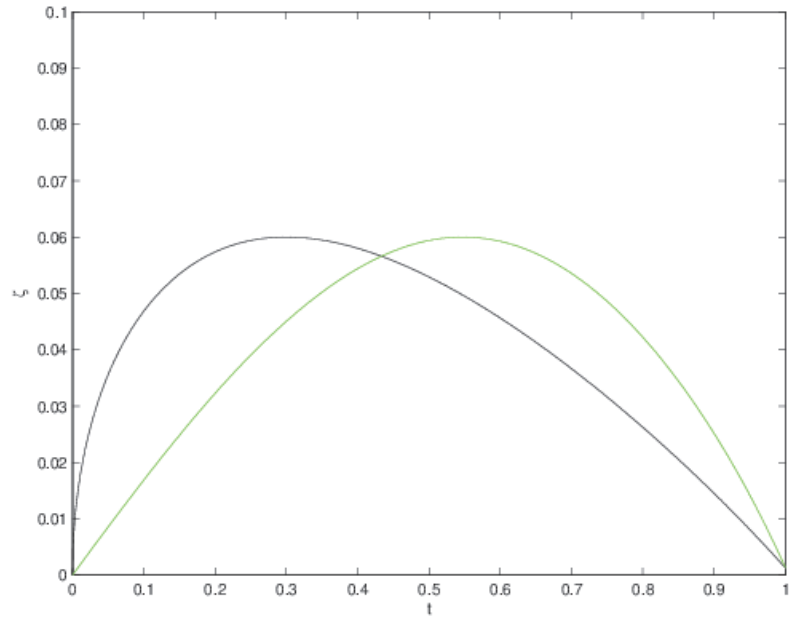

(b) $\zeta$-coordinates

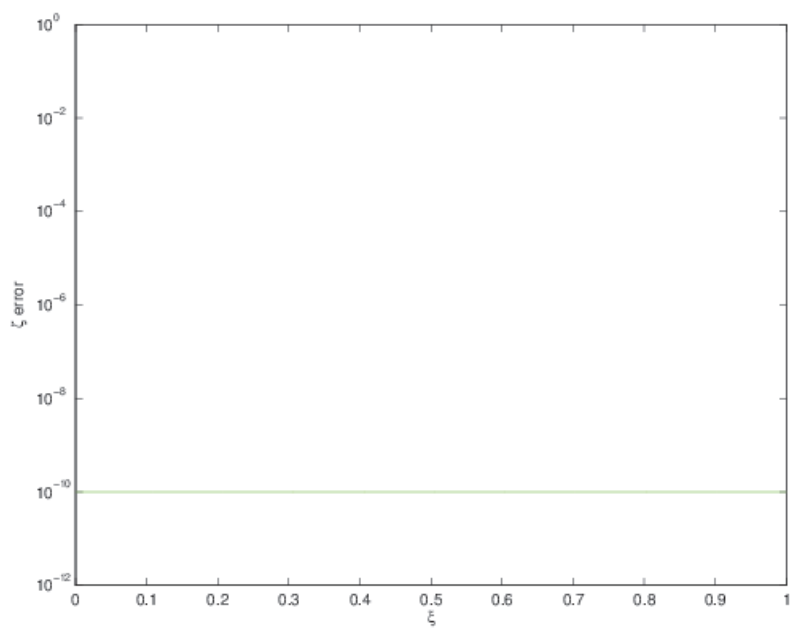

(d) Absolute Error

Figure 1. An example of a CST airfoil and the resulting Bezier curve with the corresponding error on the transformed Bezier curve. Note that a minimum threshold of $10^{-10}$ was used in the absolute error calculation.

\section{A. Exact Representations}

If the $N_{2}$ term in the CST class function is an integer, i.e. either 0 or 1 since $N_{1}$ and $N_{2}$ are bounded between those values inclusively, then the CST curve can be represented exactly as a Bezier curve. This means that geometry classes $1,3,6$ and 7 can be represented exactly using this technique. Geometry class 1 is the airfoil case from above.

\section{Geometry Class 3}

Geometry class 3 has the following form

$$
\zeta=\xi(1-\xi) S(\xi)+\xi \Delta \zeta_{\text {t.e. }}
$$

The combined class and shape functions will result in a polynomial of degree $n+2$ for a degree $n$ shape function. The trailing edge offset in then just a linear term that is added to the polynomial. Clearly this expression is a polynomial and thus can be converted to an explicit Bezier curve of degree $n+2$. 


\section{Geometry Class 6}

Geometry class 6 has the following form

$$
\zeta=\xi S(\xi)+\xi \Delta \zeta_{\text {t.e. }}
$$

which again results in a polynomial. This time the combination of the class and shape functions yields a degree $n+1$ polynomial, and the trailing edge offset again is a linear term added to the polynomial. The resulting polynomial can then easily be transformed into an explicit Bezier curve.

\section{Geometry Class 7}

Geometry class 7 has the following form

$$
\zeta=S(\xi)+\xi \Delta \zeta_{\text {t.e. }}
$$

which, similarly to the other cases, can easily be converted into a degree $n$ explicit Bezier curve.

\section{B. Approximate Representations}

The three remaining geometry classes: 2, 4 and 5, have a non-integer $N_{2}$ term which makes the preceding process of transformation not possible. Instead, a Taylor series approximation for the class function is used to develop an approximate polynomial representation.

For Geometry class 2, the CST curve representation is

$$
\zeta=\sqrt{\xi} \sqrt{1-\xi} S(\xi)+\xi \Delta \zeta_{\text {t.e. }}
$$

Converting these to a pair of parametric equations, converting the shape function to standard polynomial form and performing the $t=s^{2}$ transformation as done above yields

$$
\begin{aligned}
& \xi=s^{2} \\
& \zeta=\sqrt{1-s^{2}} \sum_{i=0}^{n} a_{i} s^{2 i+1}+s^{2} \Delta \zeta_{\text {t.e. }}
\end{aligned}
$$

The leading $\sqrt{1-s^{2}}$ term is not a polynomial, however it can be expressed as a binomial series, which is convergent over the entire interval of $s \in[0,1]$, and has the following form, see any standard calculus book such as ${ }^{4}$

$$
\sqrt{1-s^{2}}=\sum_{i=0}^{\infty}(-1)^{i} \quad \begin{gathered}
1 / 2 \\
i
\end{gathered} s^{2 i} \equiv \sum_{i=0}^{\infty} \alpha_{i} s^{2 i}
$$

where the generalized binomial coefficient is used. Substituting back into (18b) yields

$$
\zeta=\sum_{i=0}^{\infty} \alpha_{i} s^{2 i} \quad \sum_{i=0}^{n} a_{i} s^{2 i+1}+s^{2} \Delta \zeta_{\text {t.e. }}
$$

Truncating the infinite series to the first $m$ terms yields into (20) yields

$$
\zeta=\sum_{i=0}^{m} \alpha_{i} s^{2 i} \quad \sum_{i=0}^{n} a_{i} s^{2 i+1}+s^{2} \Delta \zeta_{\text {t.e. }}
$$

Unfortunately, even a Binomial series expansion of degree $5, m=5$, yields an unacceptably large error over the majority of the parameter space with the maximum error occurring at $s=1$ of 0.25 . Figure 2 shows the results up to $m=5$. While the series is absolutely convergent, the rate of convergence is quite slow. Also, recall that for $m=5$ the resulting polynomial will be $2 m=10$. Thus the resulting polynomial from this process will be of degree $2 m(2 n+1)$, which quickly becomes too large to be practical. 


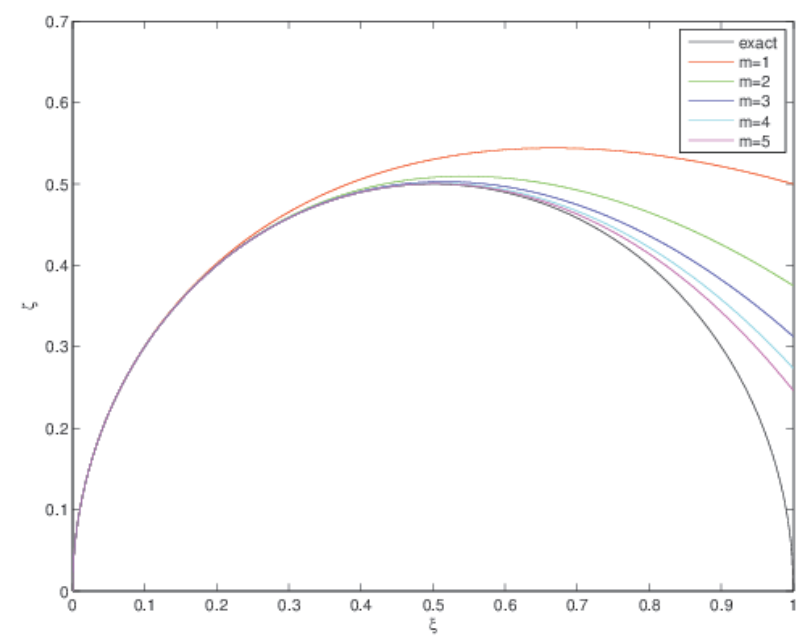

(a) Curves

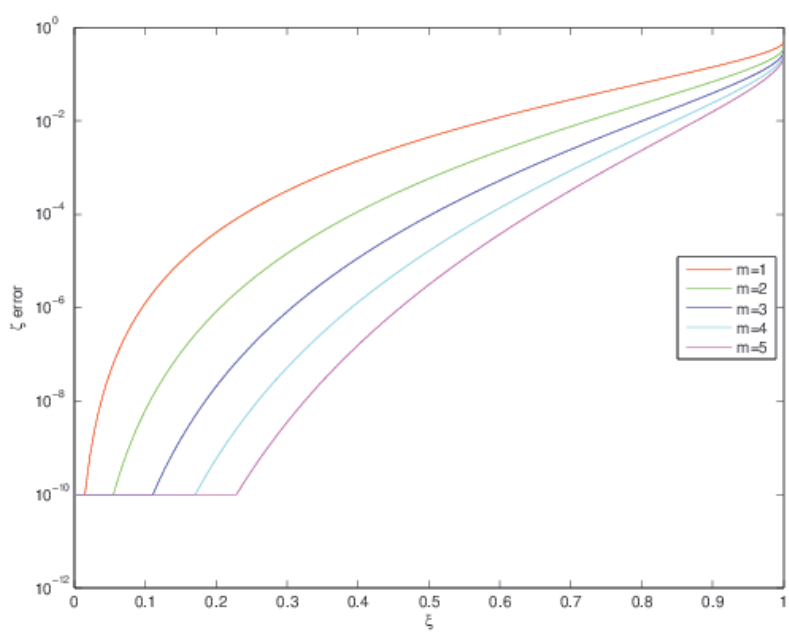

(b) Absolute Error

Figure 2. Binomial series representation of the class type 2 equation and the corresponding errors for polynomials of increasing degree using equation (19).

\section{Conclusion}

An exact conversion between the most common CST shapes to Bezier curves has been presented and shown to work to convert airfoils with only negligible error. For the cases where $N_{2}$ is not an integer, a binomial series approximation was presented that has theoretical convergence properties but has unacceptably large errors for practical uses. An alternative approach, such as using rational bezier curves, will be needed to develop a practical approximate conversion for these geometry classes.

Extending this work to three-dimensions is a trivial effort once the CST curve transformation has been accomplished. Kulfan ${ }^{1}$ presented two methods to create surfaces from CST shapes: (1) CST curves are used as cross-sections of a geometry and (2) the shape function in the curve representation is replaced with a surface shape function via Bezier surfaces in a local chord and span parametric coordinate system. Both approaches rely on using Bezier surfaces in the span-wise direction and CST curves in the chord-wise direction. The standard representation of a $n \times m$ Bezier surface is

$$
\vec{\xi}(u, v)=\sum_{i=0}^{n} \sum_{j=0}^{m} B_{i}^{n}(u) B_{j}^{m}(v) \vec{p}_{i, j}
$$

For any fixed $i$ or $j$ the resulting curve is a Bezier curve, so the conversion process means replacing the surface control points, $\vec{p}_{i, j}$, with the corresponding transformed control points, $\vec{d}_{i}$, from (13).

\section{References}

\footnotetext{
${ }^{1}$ Kulfan, B. M., "A Universal Parametric Geometry Representation Method - "CST"," $45^{\text {th }}$ AIAA Aerospace Sciences Meeting and Exhibit, AIAA, Reno, NV, January 2007, AIAA-2007-0062.

${ }^{2}$ Farin, G., Curves and Surfaces for CAGD: A Practical Guide, The Morgan Kaufmann Series in Computer Graphics and Geometric Modeling, Morgan Kaufmann Publishers, Inc., San Francisco, CA, 5th ed., 2002.

${ }^{3}$ Piegl, L. and Tiller, W., The NURBS Book, Monographs in Visual Communication, Springer, New York, 2 nd ed., 1997.

${ }^{4}$ Hurley, J. F., Calculus, Wadsworth Publishing, Belmont, CA, 1987.
} 